\title{
Modified Gutenberg-Richter Coefficient for Damage Evaluation in Reinforced Concrete Structures Subjected to Seismic Simulations on a Shaking Table
}

\author{
Francisco Sagasta Amadeo Benavent Climent Tomás Fernández Quirante Antolino Gallego
}

\begin{abstract}
1 Introduction
Reinforced concrete (RC) structures located in earthquakeprone areas are susceptible to suffering damage caused by the cyclic loading induced by ground acceleration during seismic events. It is well known that even moderate tremors, which may occur several times during the lifetime of a structure, produce cumulative damage to concrete. This damage is due to the opening of new cracks or the growth of previous ones, slippage, or even the yielding of reinforced bars in concrete.

For this reason, it is strongly desirable to establish nondestructive inspection methods to evaluate the deterioration of concrete structures quantitatively in early stages. The acoustic emission (AE) technique has been proven as a reliable method to monitor the formation and growth of cracks in concrete at the material and structural level [1-9].
\end{abstract}

In analyzing AE data acquired during tests, parameter analysis is widely employed. One example is the $\mathrm{AE}$ peak amplitude, a parameter closely related to the magnitude of fracture. Of particular significance is the $b$-value, obtained from the amplitude distribution of $\mathrm{AE}$ data. This parameter is computed on the basis of the power-low relation between the amplitude of AE events and their frequency, applying the Gutenberg-Richter relationship [10], modified for the AE technique in terms of the peak amplitude in AE decibels. This index was applied in the past for assessing the damage of reinforced concrete beams subjected to (static) cyclic loading $[11,12]$. Other applications include the health monitoring of retrofitted RC structures [13] and the evolution of cracks in concrete and cement mortar [14]; these studies suggest a limit $b$-value that determines the transition from micro-crack growth to macro-crack formation in concrete. Accordingly, macro-cracks start to develop when the $b$-value is less than 0.05. Later, the technique for calculating the $b$ value was modified by Shiotani and collaborators, who incor- 
porated statistical values of amplitude distribution analysis and defined the so-called improved $b$-value, or $i b$-value. This index has been evaluated from the AE recorded in uniaxial (static) compression tests on granite, rock, and concrete [15$18]$. Both the $b$-value and the $i b$-value were properly compared during rock fracture by Rao and collaborators [18].

The interpretation of the $b$-value and the $i b$-value in relation with the development of macro-cracks in concrete has only been based on static and quasi-static (cyclic) tests, however. The validity of this limit in the case of dynamic earthquake-type loading has not yet been addressed, and it stands as the main purpose of this paper.

More specifically, the $b$-value and $i b$-value were calculated here using the AE signals measured during several dynamic tests carried out on an RC frame structure with the shake-table of the Laboratory of Dynamics of Structures of the University of Granada. Prior to calculating the $b$-value and $i b$-value, some procedures were applied to separate as much as possible the $\mathrm{AE}$ signals coming from concrete cracking (primary sources) and the spurious AE signals (secondary sources) coming from other mechanisms-e.g. the movement of the shake table, the friction between the test specimen and the shake table, or friction between the test specimen and different parts of the experimental set-up. The presence of a large amount of such spurious AE signals is actually the main difference between the AE measurements obtained from dynamic and from static or quasi statics tests. These spurious signals can make data analysis with the AE technique very challenging. In this study a filtering procedure is used for pre-processing the $\mathrm{AE}$ signals, for a suitable separation between pertinent and not pertinent signals. It is based on the use of the Root Mean Square (RMS) in several temporal windows as the signal feature. Moreover, guard sensors were placed on parts of the test model where friction was clearly being generated.

After filtering, by comparing the $b$ - and $i b$-values with the actual damage observed in the $\mathrm{RC}$ frame, it was found that the 0.05 limit of the $b$-value that previous research associates with the onset of severe damage (i.e. development and growth of macro-cracks in concrete) should be revised for dynamic earthquake-type loadings. The validity of the tentative 0.04 value is supported by the actual (qualitative) damage observed by the naked eye during the tests, and by the (quantitative) damage measured with the well-known inter-story drift index (IDI) [19].

\section{Test Model, Experimental Set-Up and Instrumentation}

An RC frame sub-structure consisting of four columns and two beams connected by rigid joints was designed and built at the Laboratory of Dynamics of Structures of the Univer- sity of Granada (see Fig. 1). Figure 1 left shows the names assigned to the columns: $\mathrm{C} 1, \mathrm{C} 2, \mathrm{C} 3$ and $\mathrm{C} 4$. The connection of each column- $\mathrm{C} 1, \mathrm{C} 2, \mathrm{C} 3$ and $\mathrm{C} 4-$ with the beam will be referred to hereafter as the beam-column connection P1, $\mathrm{P} 2, \mathrm{P} 3$ or $\mathrm{P} 4$, respectively. The connections that have beams only at one side (right side) of the column (i.e. P1 and P3) will be called "exterior connections", and those with beams at both sides (i.e. P2 and P4) will be referred to as "interior connections". The test specimen was designed following the current provisions of the Eurocode8 [20] and the Spanish seismic code [21], assuming location in Granada (Spain). The concrete compressive strength assumed in calculations was $25 \mathrm{MPa}$, and the yield strength of the reinforcing steel was $500 \mathrm{MPa}$. The test model was designed following modern codes to develop "a strong column-weak beam" mechanism under lateral loading. A detailed description of the test specimen and design criteria can be found in reference [19].

The test specimen was subjected to five seismic simulations, referred to as $\mathrm{C} 50, \mathrm{C} 50 \mathrm{~B}, \mathrm{C} 100, \mathrm{C} 200$ and $\mathrm{C} 300$ hereafter, with the uniaxial MTS $3 \times 3 \mathrm{~m}^{2}$ shake-table of the University of Granada. In each seismic simulation, the shake-table was set to reproduce the ground motion recorded at Calitri station (Italy) during the Campano Lucano (1980) earthquake, respectively scaled in acceleration amplitude to $50,50,100,200$, and $300 \%$. The corresponding peak ground accelerations, PGAs, were $0.08,0.08,0.16,0.31$ and $0.47 \mathrm{~g}$. Each PGA represents a different seismic hazard level (SHL) at the site (Granada) that will be referred to hereafter as SHL1, SHL-1B, SHL-2, SHL-3 and SHL-4 respectively. SHL-1 and SHL-1B represent a "very frequent" earthquake, SHL2 a "frequent" earthquake, SHL-3 a "rare" earthquake, and SHL-4 a "very rare" earthquake or the "maximum considered". Figure 2 shows the history of acceleration actually measured on the shake-table during each seismic simulation.

An AMSY-5 Vallen System was used to capture the AE signals during testing. Only beam-column connections P3 and P4 were instrumented with AE sensors. Twenty VS30 AE flat low-frequency sensors were placed on the P3 and P4 beam-column connections, at the twenty positions indicated in Fig. 1a, b. These sensors were set in the range $20-80 \mathrm{kHz}$, using the $25-180 \mathrm{kHz}$ frequency band during signal acquisition with a sample period of $0.4 \mu$ s and 2,048 data for recording waveforms (200 of them as pre-triggering). Thus, the entire duration of the record window was $t_{\max }=819.2 \mu \mathrm{s}$. During acquisition, $34 \mathrm{~dB}$ gain preamplifiers and a $50 \mathrm{~dB}$ detection threshold were used. Metallic supports and silicone grease were used to fix the sensors.

AE events were created with the Event Builder application of commercial VisualAE software. More precisely, for the exterior beam-column connection, sensors S3, S7 and S8 were configured as normal ones, programming the rest of the sensors as guards. For the exterior beam-column connection, S4, S6, S9 and S10 were the normal sensors, the rest being 

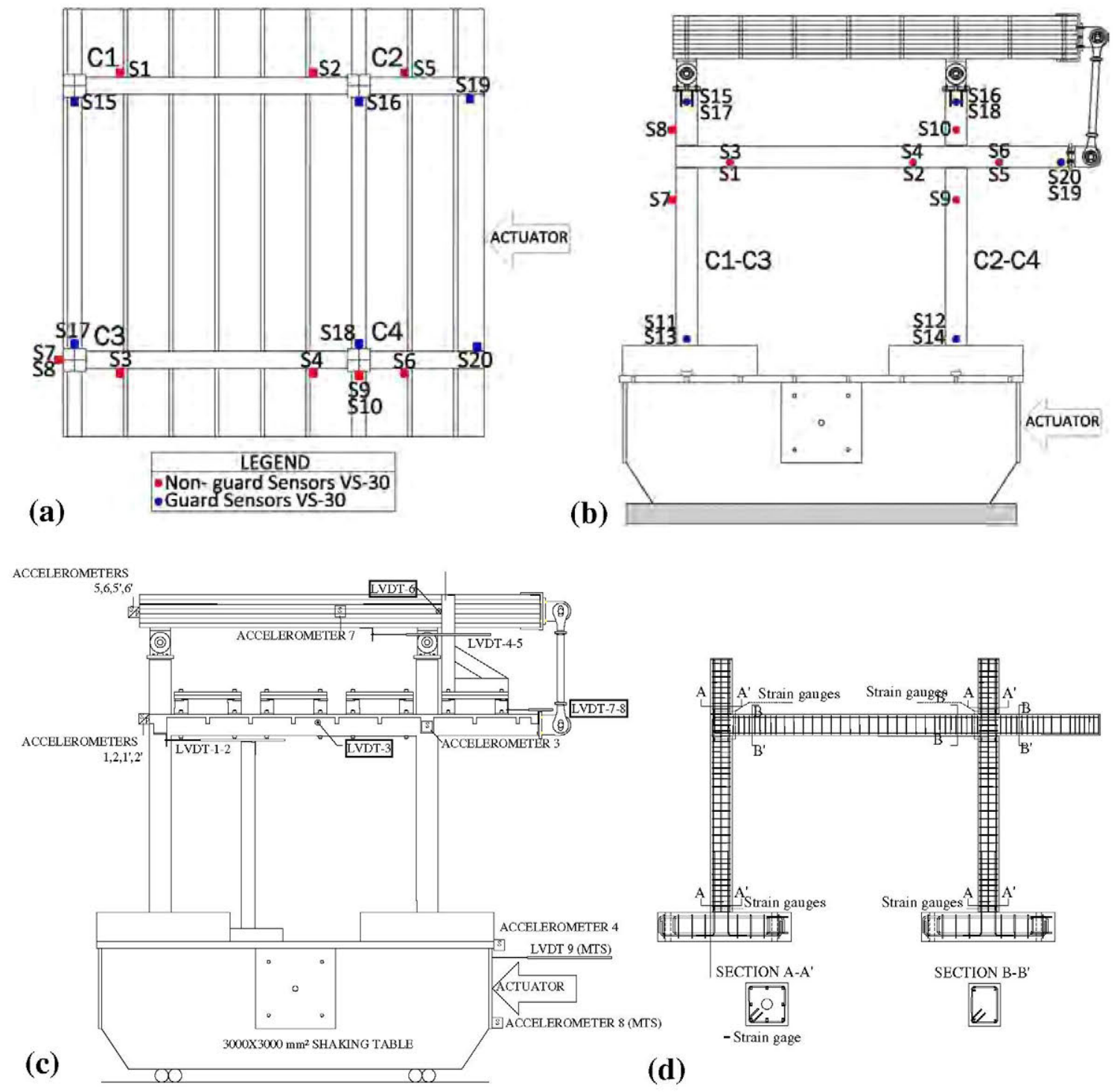

Fig. 1 Test model. AE sensor positions in bottom view plan (a) and elevation (b). Locations of accelerometers and displacement transducers (c) and location of strain gauges (d)

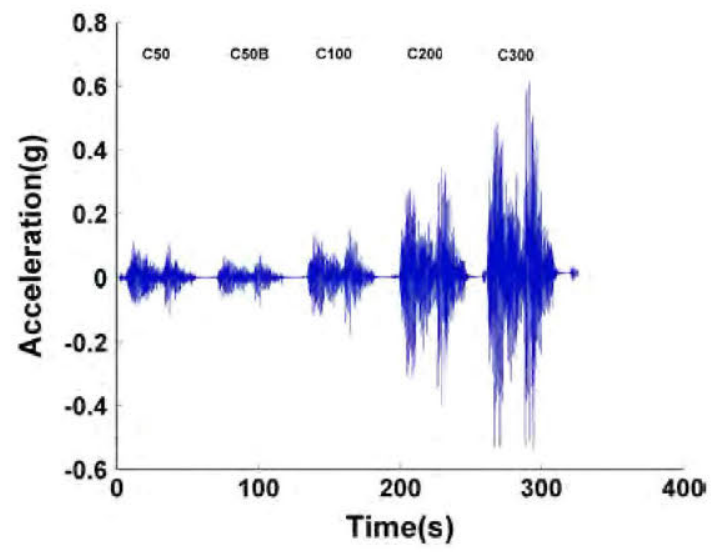

Fig. 2 Acceleration of the seismic events of different intensity applied to the test model considered as guards. Thus, only those events whose first-hit corresponded to normal sensors were considered, limiting $\mathrm{AE}$ monitoring to the local area of each beam-column connection.

The specimen was instrumented with 12 uniaxial accelerometers and 9 displacement transducers (linear variable differential transformers, LVDTs) as shown in Fig. 1c, and 192 strain gauges as shown in Fig. 1d. Displacement transducers 1, 2, 4, 5, 7, 8 and 9 measured the in-plane translations and the inter-story drifts in the direction of the seismic loading. Displacement transducers 3 and 6 measured translations in the direction perpendicular to the seismic loading. Accelerometers 1, 2, 14', 2', 4, 5, 6, 5', 6' and 8 measured accelerations in the direction of the seismic loading, while accelerometers 3 and 7 provided the accelerations in the 
direction perpendicular to the seismic loading. Accelerometers 1 and $1^{\prime}, 2$ and $2^{\prime}, 5$ and $5^{\prime}, 6$ and $6^{\prime}$ were placed in the same position but had different sensitivity. Strain gauges were attached to the surface of longitudinal reinforcement when construction was in progress; they were located at column and beam ends. These data were acquired continuously with a scan frequency of $200 \mathrm{~Hz}$.

\section{Ae Signal Filtering Procedure}

Previous identification of undesirable mechanical noise sources in the specimen was carried out. As the first action, to prevent friction noise generated between the different metallic elements located in the specimen (added steel blocks, screws, fixing systems of sensors, accelerometers, LVDTs,

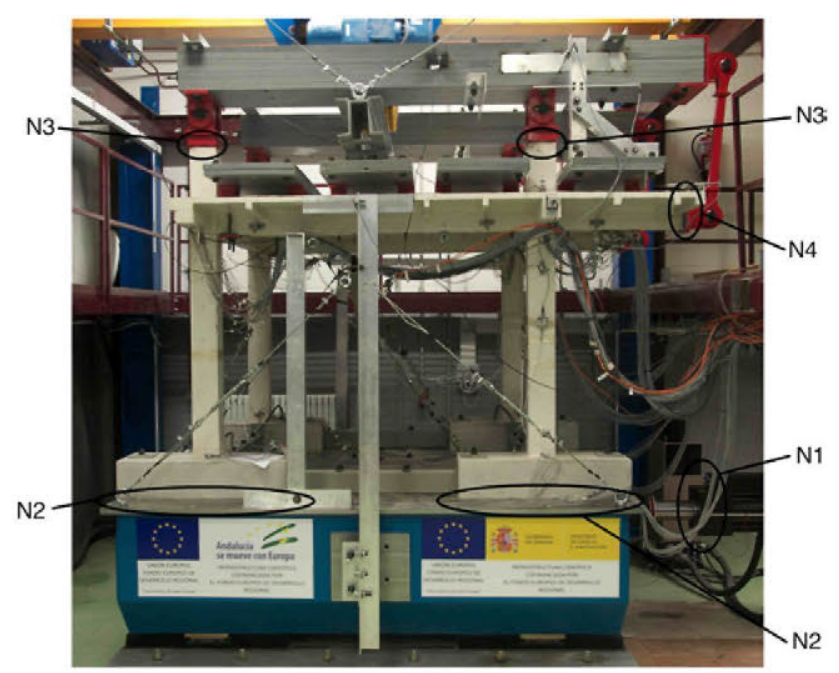

Fig. 3 Mechanical noise sources N1-N4 foreseen in the AE test

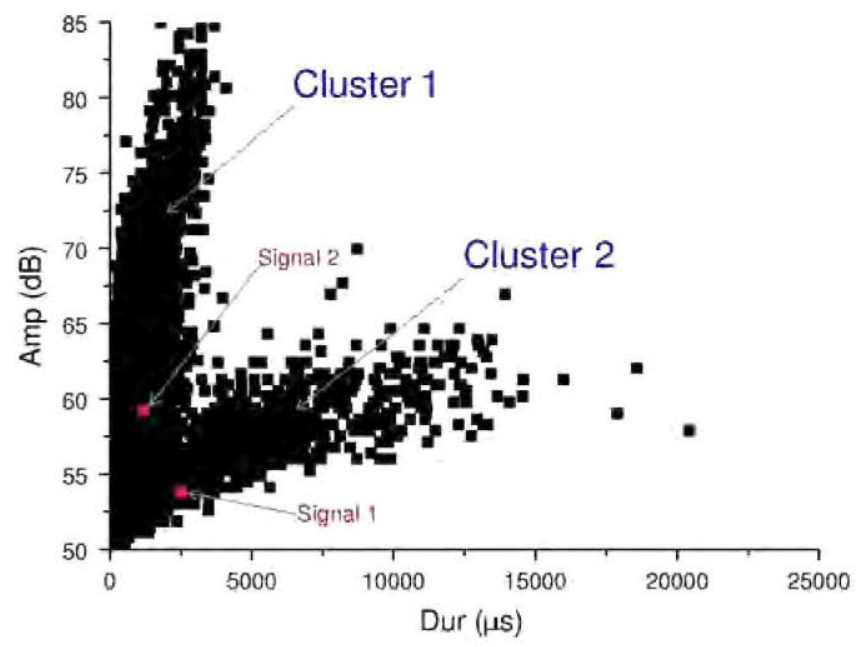

etc.), rubber and Teflon films were inserted between any two contacting surfaces susceptible of generating noise. Still, other important noise sources were observed (indicated in Fig. 3):

- Noise source 1 (N1) Remarkable mechanical continuous noise coming from the oil flow in the actuator of the shaketable was detected. The level of this noise was above $120 \mathrm{~dB}$ in the actuator and $70 \mathrm{~dB}$ at the base of the columns, reaching most of the $\mathrm{AE}$ sensors with amplitudes varying from 60 to $30 \mathrm{~dB}$. This noise produced mainly continuous AE signals in all twenty sensors placed on the specimen.

- Noise source 2 (N2) Friction noise generated on the surface where the foundation of the columns was in contact with the shake-table.

- Noise source 3 (N3) Friction noise generated on the surface where the upper end of the columns was in contact with the pin joints supporting the inertial mass.

- Noise source 4(N4) Friction noise generated on the surface where the beam-end was in contact with the pin joints assembling the vertical steel struts with the inertial mass.

Noise coming from sources N1 and N2 was prevented by means of guard sensors located on the base of the columns (sensors S11-S14). In the same way, noise coming from sources N3 and N4 was prevented with guard sensors S15S17, and S19-S20, respectively.

As an example, Fig. 4 left shows an Amplitude-Duration diagram of all the events recorded during the seismic simulation C50, whatever the first-hit sensor was. Two clusters can be clearly observed (Clusters 1 and 2). Specifically, signals from Cluster 2 have very high values of duration and not very high amplitudes, suggesting their correspondence with the noise source N1. Figure 4 right shows the same

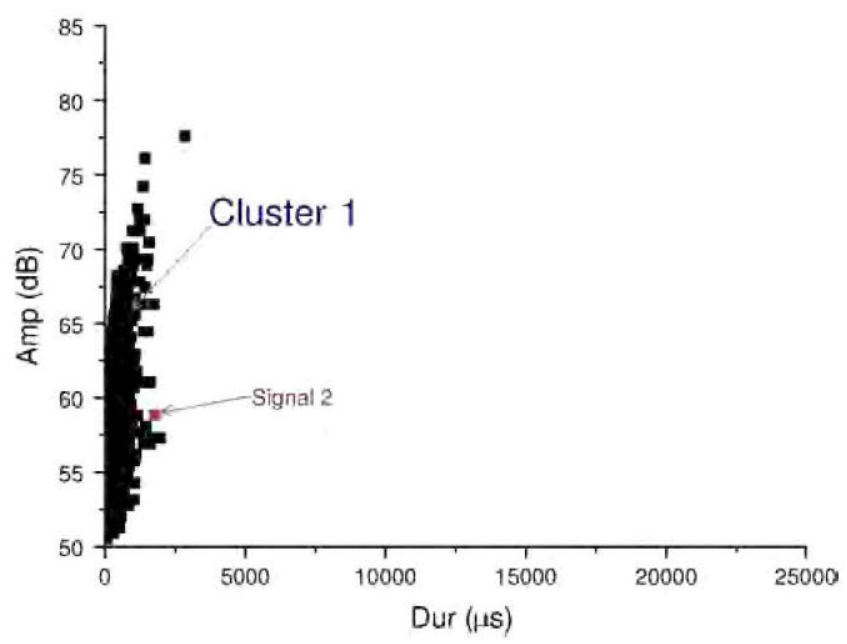

Fig. 4 Amplitude versus duration of the events recorded during simulation C50. Left before applying guard-sensors filter. Right after applying guard-sensors filter 

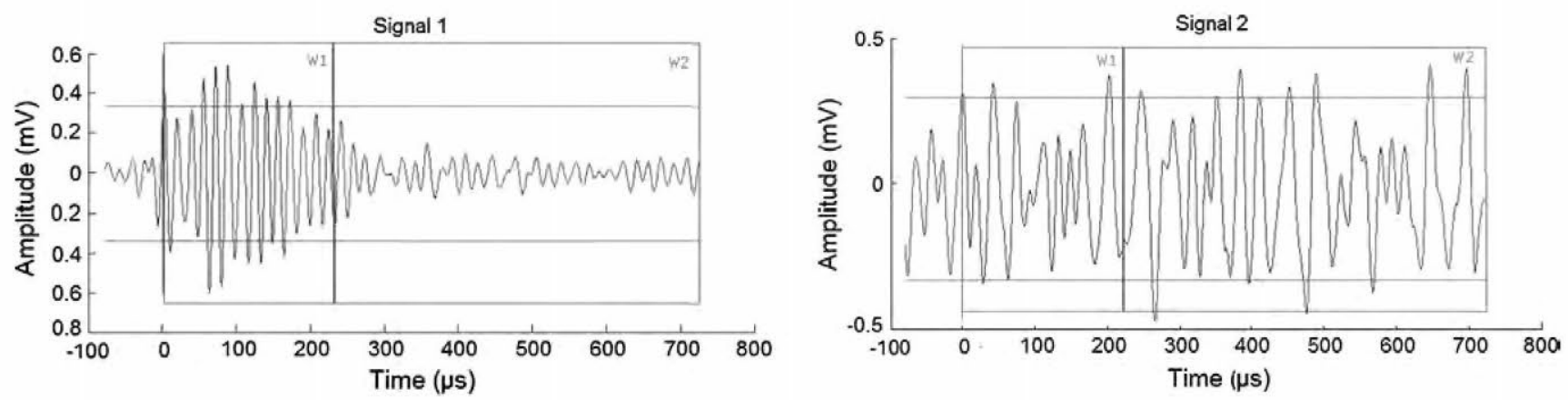

Fig. 5 Some AE Signals corresponding to seismic simulation C50. Left Type (i); Right Type (ii). Windows W1 and W2 are marked on the signals
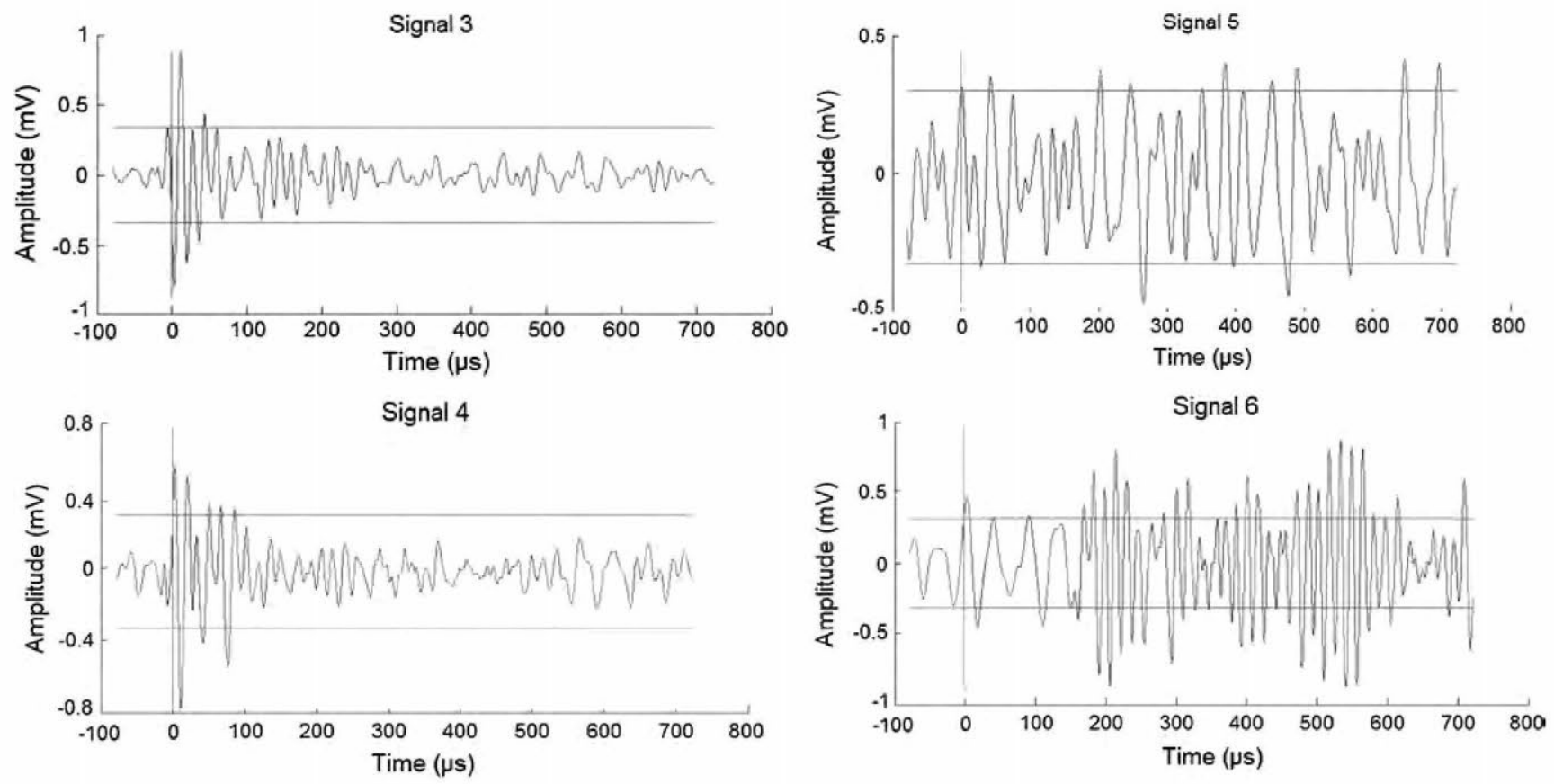

Fig. 6 Left column Type (i) signals; right column: Type (ii) signals

diagram after configuring the guard sensors as mentioned before. It can be clearly seen that most events from Cluster 2 have been filtered, sustaining their probable association with noise source N1. It is also possible that some of them came from noise sources N2-N4. Furthermore, the number of signals in Cluster 1 is clearly seen to diminish, most likely due to the elimination of some signals coming from noise sources N1-N4 by the guard-sensors filter.

Yet even after applying the guard-sensor filtering, many continuous signals were observed. Figure 5 right shows one of them (Signal 2). Such signals could be traced to different mechanisms: (i) those associated with noise sources N1$\mathrm{N} 4$, which were not filtered by the guard-sensor; (ii) interior friction from existing cracks in the concrete; (iii) shearcracks producing large waveforms $[22,23]$. Moreover, the propagation from the event location to the sensor makes the signal longer, distributing the energy throughout its duration.

Thus, additional filtering was necessary to separate these $\mathrm{AE}$ signals from the genuine ones coming from concrete cracking.

Detailed observation of the $\mathrm{AE}$ waveforms that passed the guard filtering showed that these signals could be divided into two qualitatively different groups:

Type (i) short-duration signals (burst signals), whose energy was concentrated mainly at the beginning of the signal, and whose duration was not excessively high.

Type (ii) long-duration signals (continuous signals), whose energy was not concentrated at the beginning of the signal, but distributed along the whole signal. 
Fig. 7 AE cumulative frequency-amplitude distribution corresponding to different test times. Left exterior beam-column connection P3. Right interior beam-column connection P4
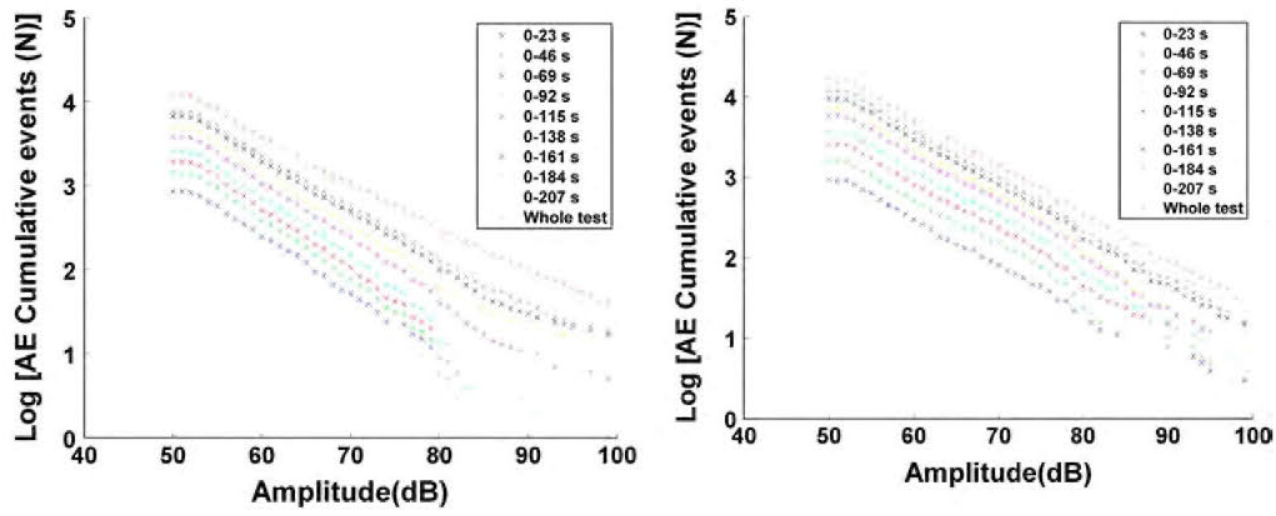

Based on past research, it was assumed that the shortduration and high-amplitude signals referred to above as Type (i) would correspond to concrete cracking. As an example, Fig. 5 left and right represent Type (i) and Type (ii) signals, respectively.

In order to separate the two kinds of signals, we applied a filtering procedure based on the RMS (Root Mean Square) of the signal calculated in time windows $\mathrm{W} 1$ and W2, as marked in Fig. 5. Basically, those signals for which $R M S_{W 1}>$ $R M S_{W 2}$ were associated with Type (i). As an example, Fig. 6 shows two signals classified as Type (i) (Signals 3 and 4 ) and two signals classified as Type (ii) (Signals 5 and 6 ) by means of this filter. Simple visual observation confirms that all of them were correctly classified. It is also remarkable that most of the Type (ii) signals appear to belong to Cluster 2, near the intersection with Cluster 1.

It should also be underlined, however, that even when this RMS filter eliminated the remaining signals associated with friction or with sources N1-N4 in an effective way, it probably also removed some genuine signals coming from concrete cracking modes and waveforms originated by concrete cracking that were strongly distorted over the travels from source to sensor. Furthermore, given the statistical nature of the AE method, filtering is usually not $100 \%$ effective.

The length of $\mathrm{W}_{1}$ was established in view of physical criteria using the propagation speed of longitudinal waves in a bar of this kind of concrete, and the maximum distance between the center of the structure and the sensors. More details regarding this filter can be found in reference [24].

\section{$4 b$ and $i b$-Value Calculation}

\section{$4.1 b$-Value}

In seismology the well-known Gutenberg-Richter law establishes that [10]

$\log _{10} N=a-b M$, where $N$ is the total number of earthquakes with magnitude higher than $M$ in any given region and period of time; $a$ is an empirical constant; and $b$ is the well-known $b$-value, defining the slope of the linear relationship given by Eq. (1). This law has been adapted to the $\mathrm{AE}$ signals measured during a given period of time in a material that fractures under a given loading as [11]

$\log _{10} N=a-b\left(\frac{A_{d B}}{20}\right)$,

where $A_{d B}$ is the peak amplitude of the AE signals measured in decibels and $N$ is the number of $\mathrm{AE}$ events with amplitudes higher than $A_{d B}$, measured during the considered period of time. Taking into account that the AE peak amplitude is directly related with the magnitude of fracture, the $b$-value, defined in Eq. (2) as the slope of the AE peak amplitude distribution, has proven to be an effective index to characterize the formation and growth of cracks during the time period considered. Indeed, this is a global parameter appropriate for characterizing signals of stochastic processes such as earthquakes or $\mathrm{AE}$ signals. According to previous work [11-14], the $b$-value calculated at successive time windows of the loading process changes systematically and hence can be used to study the development of the cracking process. For a given time period of observation, high $b$-values indicate the occurrence of a large number of small-amplitude $\mathrm{AE}$ hits, associated with micro-crack formation and slow crack growth. In contrast, low $b$-values are associated with macro-crack formation and faster growth. The latter (i.e. the fast development of macro-cracks) involves much more damage on the structural elements than the former (i.e. the slow development of micro-cracks). Past research [11-18] established $b=0.05$ as the boundary value between slow microcrack and fast macro-crack formation. Note that the $b$-value is divided by 20 for comparison with the $i b$-value. We should underline that the limit $b=0.05$ was obtained from static and pseudo-static tests. Its validity for realistic earthquaketype dynamic loadings is examined in this paper, as explained below. It should also be stressed that the $b$-value provides a 
Fig. 8 b-value: left exterior beam-column connection P3. Right interior beam-column connection $\mathrm{P} 4$. The $b$-value is divided by 20 for comparison with $i b$-value
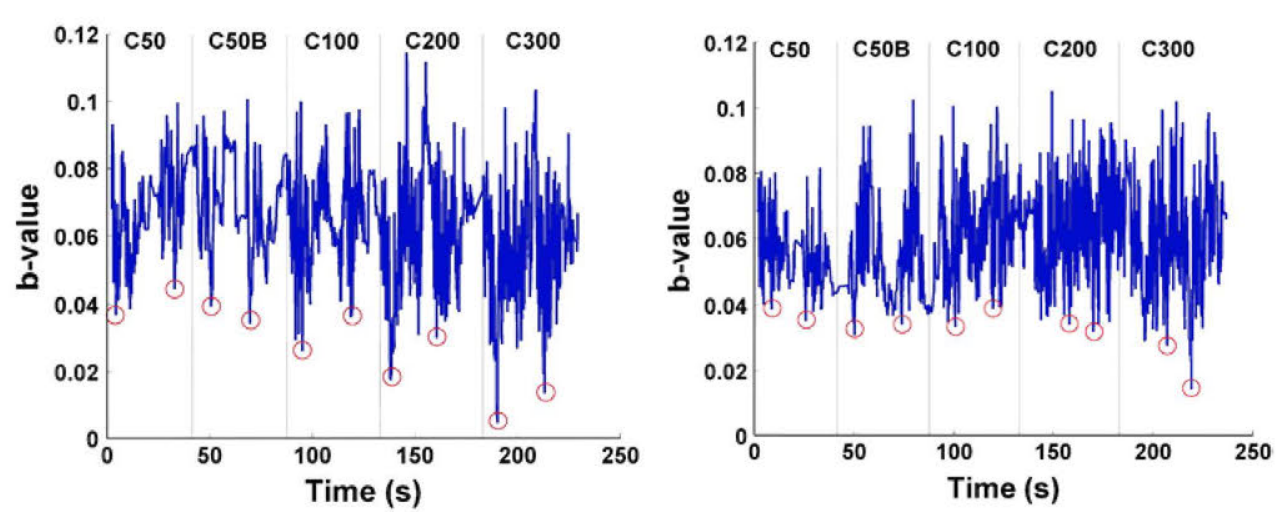

Fig. 9 Correlation coefficients for different values of $\beta$. Left exterior beam-column connection P3. Right interior beam-column connection $\mathrm{P} 4$
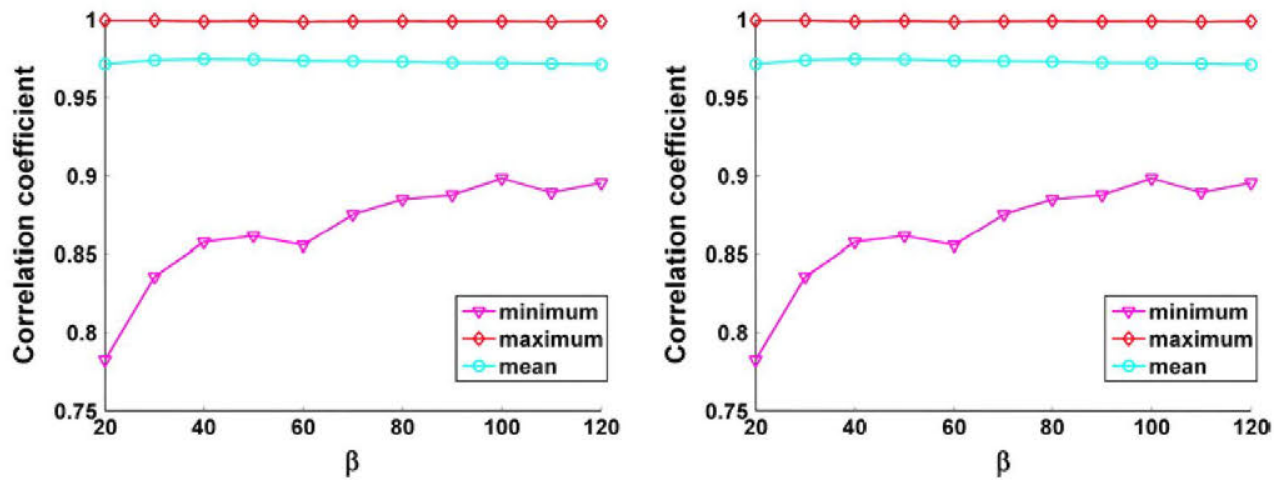

snapshot of the cracking process, that is, insight regarding the damage occurring in the time period considered, but it does not provide information on the accumulated damage of the structure.

A key aspect in the calculation of the $b$-value is the selection of the range of $\mathrm{AE}$ amplitudes in which the relation between $N$ and the AE amplitude is approximately linear, since very often deviations from linearity occur in both the low and high AE amplitude ranges. Figure 7 shows the $N$ versus $\mathrm{AE}$ amplitude plots for beam-column connections $\mathrm{P} 3$ and $\mathrm{P} 4$, calculated at different instants $t$ (each one drawn in a different color) of the seismic test. To obtain the AE cumulativeevents curve corresponding to a given instant $t$, the 60 events measured before this instant were considered. Very good linearity is observed in Fig. 7 for each $t$.

For both beam-column connections, P3 and P4, Fig. 8 shows the $b$-value obtained at each instant $t$ from the onset of the first seismic simulation C50 to the end of the last one, C300. According to Eq. 2, the $b$-value at a particular instant $t$ was calculated using the last sixty $\mathrm{AE}$ signals recorded before this instant $t$, i.e. using a population data of 60 . The slope (i.e. $b$-value) of $N$ versus the peak amplitude was calculated by means of a linear least squares fitting. It can be observed that the $b$-value oscillates, with peaks and valleys that can be associated with instants of slow micro-cracking (low damage generation) and fast macro-cracking (high damage generation), respectively. This behavior is to be expected, since the loading is cyclic and the acceleration $\ddot{u}_{g}$ applied to the shake-table oscillates. However, if a group of instants $t$ corresponding to increasing values of $\ddot{u}_{g}$ are selected (indicated in Fig. 8 with circles), the trend of their $b$-values is downward.

Similarly, a quick look at these figures would suggest that the exterior beam-column connection P3 suffered more severe damage than the $\mathrm{P} 4$ interior connection. Also evident is that values lower than 0.05 , correlated with macro-cracks in view of the criterion established in [15-17], appear from the very beginning of the test (seismic simulation C50). However, this result is not corroborated by means of other damage indexes and visual observation, suggesting the need to revise the limit 0.05 in the case of dynamic tests.

\section{$4.2 i b$-Value}

As mentioned in the Introduction, a modified $b$-value, referred to as the improved $i b$-value hereafter, was proposed by Shiotani and collaborators in [15-17]. Accordingly, the $i b$-value is calculated from a constant number of data points $\beta$, the number of population data. Values of $\beta$ from 50 to 100 have been previously suggested [15-17]. A value of $\beta$ is considered suitable when the relationship between $\log N$ and $\mathrm{AE}$ amplitude approaches a straight line. Furthermore, the greater $\beta$ is, the better the approximation to a straight line. The level of fitting to a straight line can be mathematically evaluated by using the correlation coefficient, which is plotted in Fig. 9 for beam-column connections P3 and P4. The graphs show the minimum, the maximum, and the mean value 
Fig. $10 i b$-Value definition

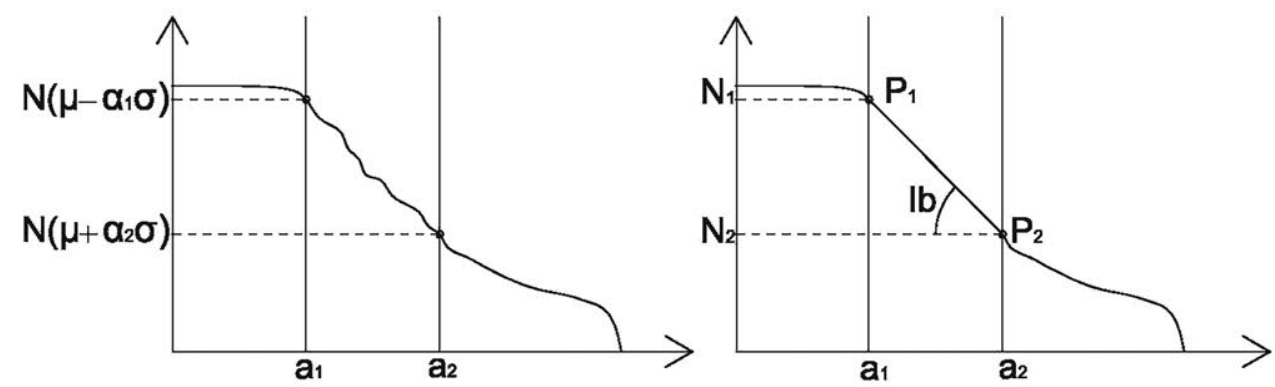

Fig. 11 Log number of $\mathrm{AE}$ events accumulated versus amplitude for three instants of seismic simulation C50 ( $t=15$, 30 and $45 \mathrm{~s})$. Left vertical line value $a_{1}$. Right vertical line value $a_{2}$. Interior beam-column joint $\mathrm{P} 3$
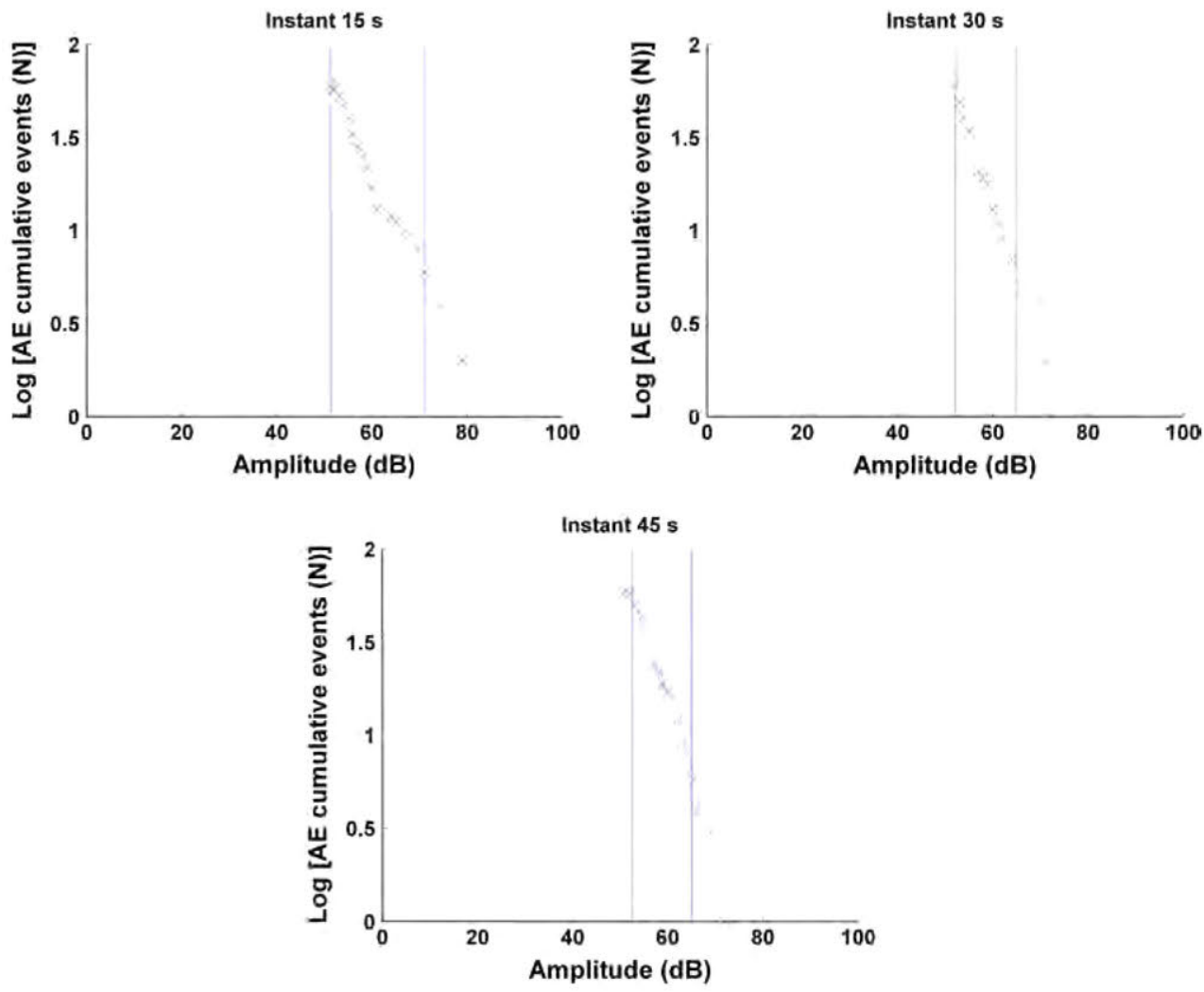

of the set of correlation coefficients obtained for each value of $\beta$. Notice that when a new AE event is recorded, the population set is updated and a new straight-line fitting and its corresponding correlation coefficient are recalculated, thus giving a set of correlation coefficients, one for each value of $\beta$. As can be seen, the mean value (statistically more representative than the minimum and the maximum values) of the correlation coefficient increases as $\beta$ increases, keeping almost constant at 0.95 from $\beta=40$. On the other hand, the linear fitting should not be the only criterion for choosing the number of points, since if many points are included the $i b$-value loses its sensitivity to transient phenomena. Taking into account both facts, $\beta=60$ was chosen as a reasonable compromise value for calculating the $i b$-value.

As already pointed out in the previous subsection, the cumulative frequency-amplitude distribution graph does not tend to a line in its entire domain of amplitudes. For this rea- son, Shiotani et al. [15-17] proposed calculating the slope ( $i b$ value) using only the data bounded by the AE amplitudes $a_{1}=\mu+\alpha_{1} \sigma$ and $a_{2}=\mu-\alpha_{2} \sigma$, as shown in Fig. 10. Here, $\mu$ and $\sigma$ are the mean and standard deviation of the $\mathrm{AE}$ amplitude distribution, respectively; and $\alpha_{1}$ and $\alpha_{2}$ are two constants established by the user in each test. Thus, the $i b$-value is obtained with

$i b=\frac{\log _{10} N\left(a_{1}\right)-\log _{10} N\left(a_{2}\right)}{\left(\alpha_{1}+\alpha_{2}\right) \sigma}$.

In this study, $\alpha_{1}$ and $\alpha_{2}$ were determined as follows. First, $\alpha_{1}$ was set to 1 for both beam-column connections P3 and P4. Figure 11 shows the $\log$ number of $\mathrm{AE}$ events accumulated in the interior beam-column connection $\mathrm{P} 4$ at several instants of the test $(t=15 \mathrm{~s}, t=30 \mathrm{~s}$ and $t=45 \mathrm{~s})$. Two vertical lines represent the amplitude range, $a_{1}$ and $a_{2}$. As is clearly seen, $\alpha_{1}=1$ provides an $\mathrm{AE}$ amplitude $a_{1}$ that approximately 
Fig. 12 Correlation coefficients for different values of $\alpha_{2}$ for all seismic simulations. Left exterior beam-column connection P3. Right interior beam-column connection $\mathrm{P} 4$
Fig. 13 Mean and standard deviation of the AE event amplitude during the five seismic simulations. Left exterior beam-column connection P3. Right interior beam-column connection P4
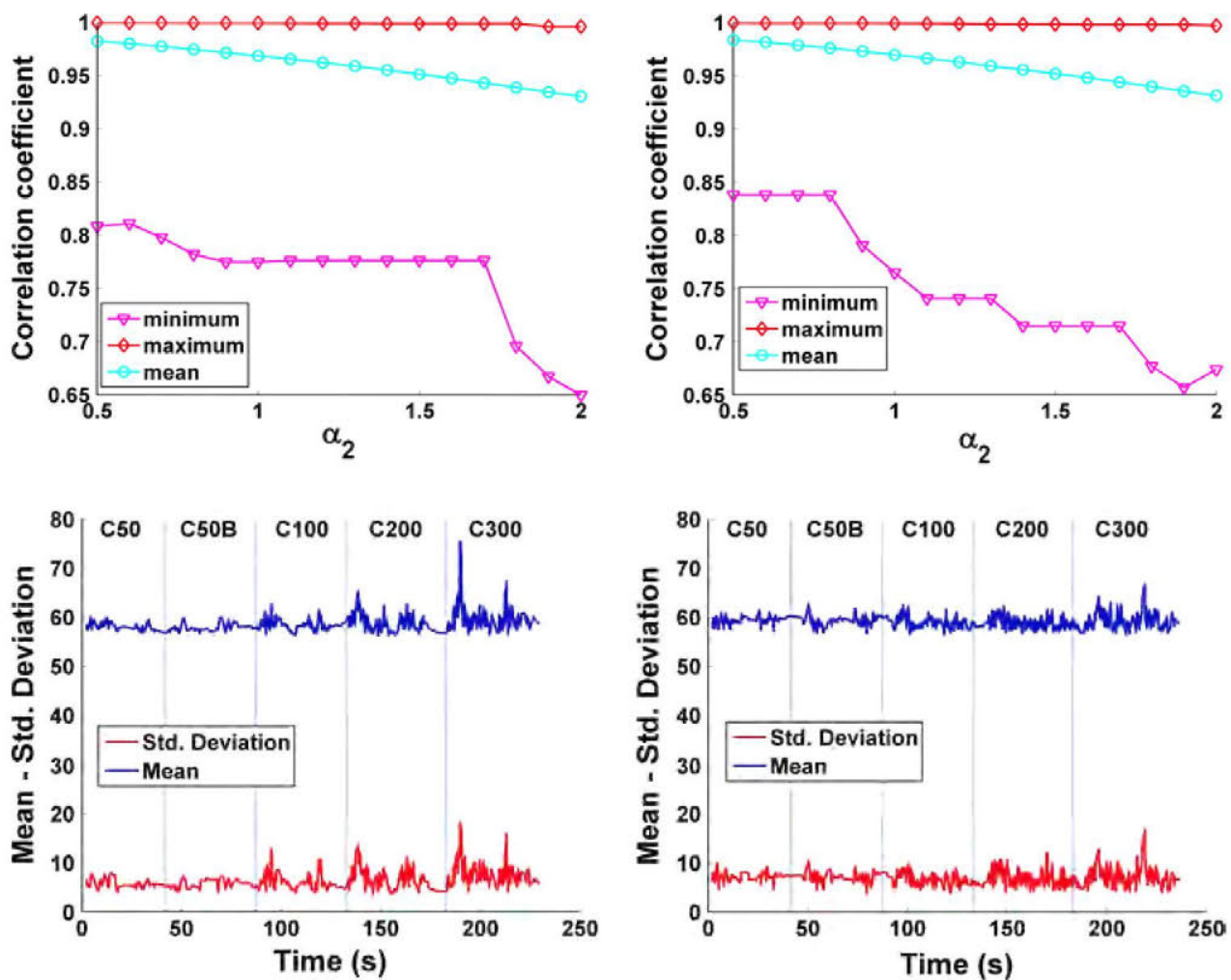

coincides with the end of the straight line. Similar results were obtained for exterior beam-column connection $\mathrm{P} 3$.

However, determining $\alpha_{2}$ was much more cumbersome because the range of $\mathrm{AE}$ amplitude corresponding to a straight line varies significantly depending on the instant $t$ and the seismic simulation considered. To make a reasonable choice of $\alpha_{2}$, the relationship between the correlation coefficient and $\alpha_{2}$ for a fixed values $\beta=60$ and $\alpha_{1}=1$ was calculated, and it is shown in Fig. 12. As expected, the correlation coefficient is seen to diminish approximately linearly with $\alpha_{2}$. In this study the value $\alpha_{2}=1.5$ was chosen, providing a correlation coefficient of about 0.95 .

For $\beta=60, \alpha_{1}=1$ and $\alpha_{2}=1.5$, and at each instant $t$, the mean and standard deviation of the AE amplitudes measured in the $\beta=60$ events occurring immediately before this instant $t$ were obtained, and are shown in Fig. 13. As manifest in Fig. 13, the mean and standard deviation of the AE amplitude remain fairly stable during the first three seismic simulations, indicating that $\mathrm{AE}$ events are within the same range of amplitudes. However, in simulations C200 and C300 some peaks of amplitudes appear, corresponding with the instants of the highest acceleration applied to the shake-table. Since the AE amplitude is an indicator on the intensity of the damage imparted to a structure at a given instant $t$, this means higher damage imparted to the structure at this instant. The peaks are much more pronounced for the exterior beam-column connection P3 than for the interior connection $\mathrm{P} 4$. However, despite expected fluctuations, in both cases the average AE amplitude remains about $60 \mathrm{~dB}$, with relatively small standard deviations (from 8 to $20 \mathrm{~dB}$ ).

For $\beta=60, \alpha_{1}=1$ and $\alpha_{2}=1.5$, Fig. 14 shows the values of parameters $a_{1}$ and $a_{2}$ defining the range of amplitudes used for $i b$-value calculation. Many similarities can be found in the evolution of $a_{1}$ and $a_{2}$ along with the mean and standard deviation shown in Fig. 13. It is clear that $a_{2}$ presents more abrupt changes than $a_{1}$, which is consistent with the linearity ranges observed in Fig. 11-that is, the upper limit is almost stable, but the lower one is highly dependent on the instant $t$ and the seismic simulation considered.

Finally, Fig. 15 shows the $i b$-value obtained for both beamcolumn connections, P3 and P4. It is clear that the general trend for $i b$-value coincides with that described in the previous subsection for the $b$ value. This also corroborates that the level of damage imparted to the structure increased along with the increase of accelerations applied to the shaketable. Furthermore, comparison of the left and right graphs of Fig. 15 makes it evident that the exterior beam-column connection P3 suffered more damage than the interior beamcolumn one, P4. More detailed discussion of this point is offered in the next section.

\section{Discussion}

As can be seen in Figs. 8 and 15, both the $b$-value and the $i b$-value decrease as the acceleration applied to the shake- 
Fig. $14 a_{1}$ and $a_{2}$ obtained for the five seismic simulations. Left exterior beam-column connection P3. Right interior beam-column connection $\mathrm{P} 4$
Fig. $15 i b$-Value. Left exterior beam-column connection P3. Right interior beam-column connection P4
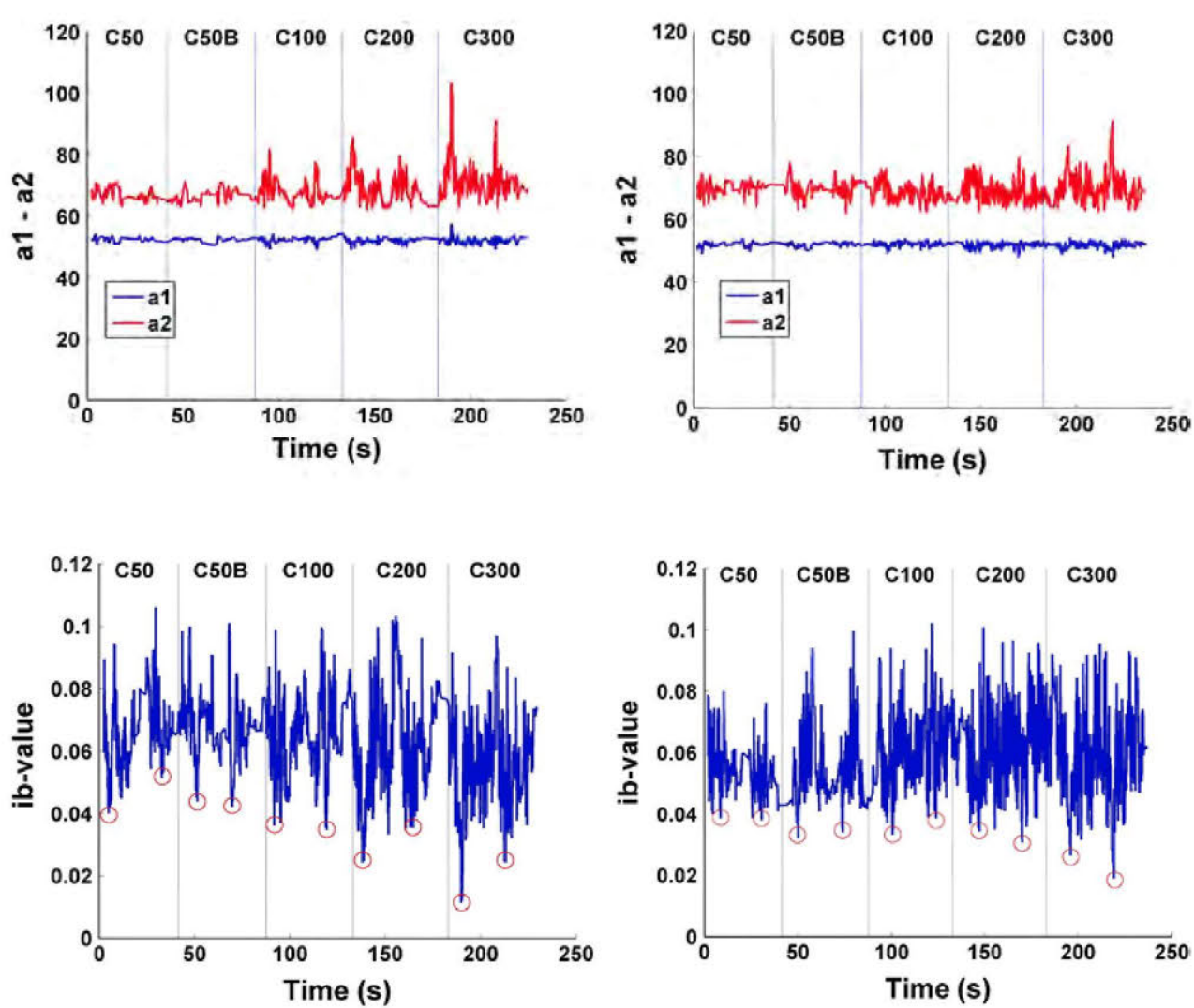

table increases. Moreover, the damage imparted to the exterior connection $\mathrm{P} 3$ appears greater than that imparted to the interior connection $\mathrm{P} 4$, since the $b$-value and $i b$-value present a more intense decrease for $\mathrm{P} 3$ than for P4 (i.e. the lower bound of both indexes is smaller for P3 than for P4). In contrast, the number of times at which the $i b$-value is lower than 0.05 (the critical value associated with the onset of macrocracks, according to previous studies based on static tests [15-17]) is larger for the interior connection P4. Accordingly, the macro-cracks in $\mathrm{P} 4$ should be more numerous than in the exterior connection P3. However, the opposite pattern was observed-that is, the number of macro-cracks in the exterior connection P3 was larger than in the interior connection P4. This contradictory result suggests that the boundary value of $i b=0.05$ associated with the onset of macro-cracks on the basis of static or quasi-static tests is not appropriate in the case of dynamic tests, meaning it should be revised for earthquake-type events. This observation is supported by the fact that, for example, $i b=0.05$ is reached many times during simulation C50 for both connections, although no macroscopic crack was visually detected. During this seismic simulation the reinforcing steel remained elastic, according to the strain measurements provided by the gauges attached to the reinforcing rebars. This fact is further corroborated by other damage indices calculated in these tests, as discussed later.

\subsection{Critical Events}

Figure 16 shows the history of acceleration applied to the shake-table during each seismic simulation. In the first row of graphs in Fig. 16, the time-acceleration points corresponding to $\mathrm{AE}$ events whose $b$-value is equal or less than 0.05 are indicated with red crosses. These $\mathrm{AE}$ events are referred to as "Critical Events" [5]. The second, third and fourth rows of graphs inside Fig. 16 show the Critical Events for several limits: $0.045,0.04$ and 0.035 . The $i b$-value is represented similarly in Fig. 17. In all cases the Critical Events are seen to be mainly located near the acceleration peaks. Moreover, as the acceleration increases, the concentration of Critical Events increases.

During the two first seismic simulations (C50 and C50B), no macro-cracks were observed with the naked eye in beamcolumn connection $\mathrm{P} 4$, which is consistent with the fact that the reinforcing rebars did not yield. However, it can be observed in Figs. 8 and 15 that the $b$-value and the $i b$-value of a significant number of Critical Events are very close to 0.05 and 0.045 , which are the values associated with the opening and growth of macro-cracks in previous studies. Since the Critical Events recorded during seismic simulations C50 and $\mathrm{C} 50 \mathrm{~B}$ do not correspond to macro-cracks, but to the creation/growth of small cracks, it would appear that the limits 0.05 and 0.045 are not appropriate for the dynamic cyclic tests 
Fig. 16 History of acceleration applied to the structure with indication (red crosses) of the critical events whose $b$-value is below a given threshold. Left exterior beam-column connection P3. Right interior beam-column connection P4 (Color figure online)
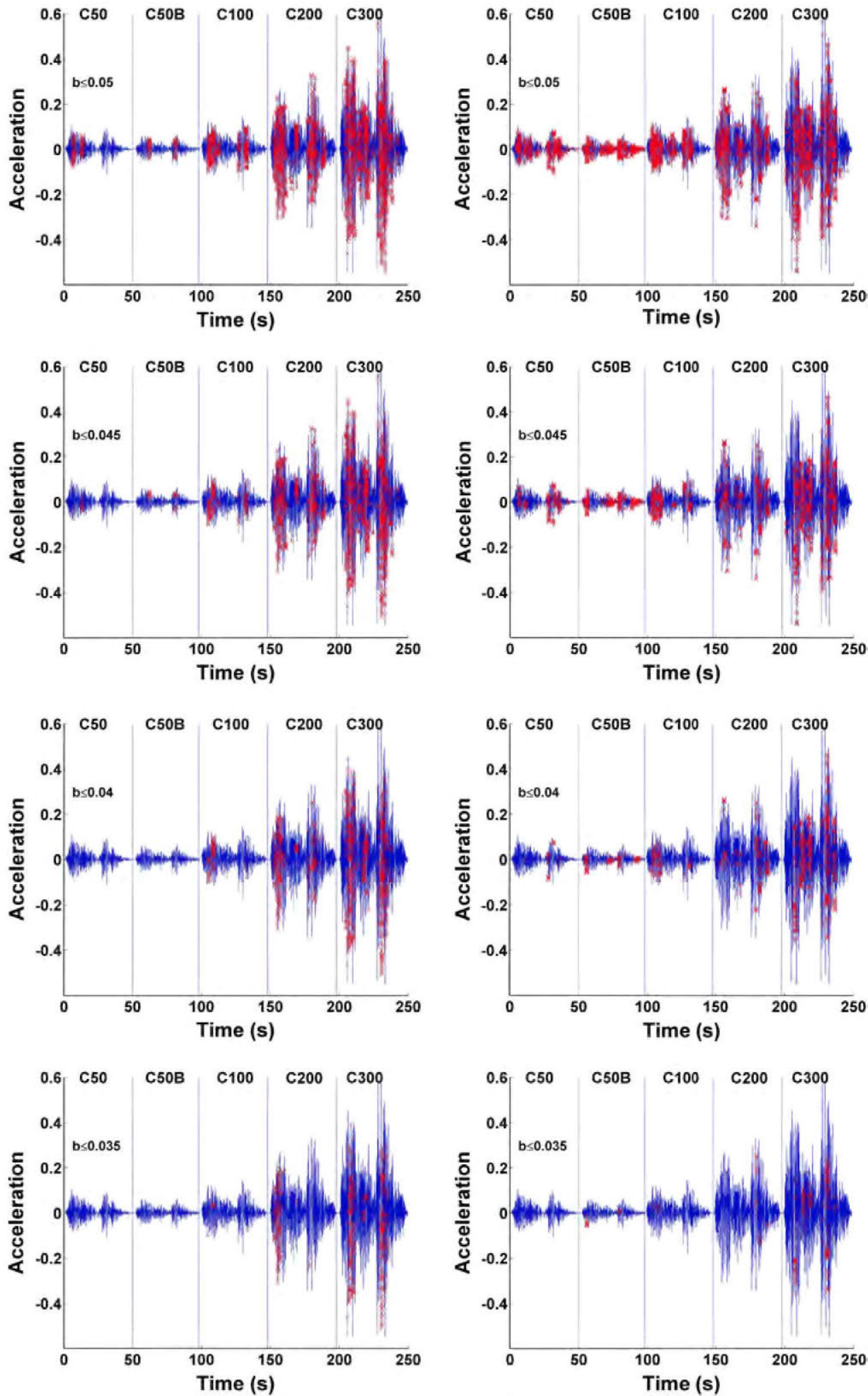

under study. Macro-cracks were visible during seismic simulations C100, C200 and C300, and Critical Events observed during these simulations had $b$ - and $i b$-values below 0.04 and 0.035 . Based on these experimental observations, the authors propose adopting 0.04 and 0.035 as the limits of $b$ and $i b$ values that characterize the formation of macro-cracks in reinforced concrete members subjected to dynamic loading. 
Fig. 17 History of acceleration applied to the structure with indication (red crosses) of the critical events whose $i b$-value is below a given threshold. Left exterior beam-column connection P3. Right interior beam-column connection P4 (Color figure online)
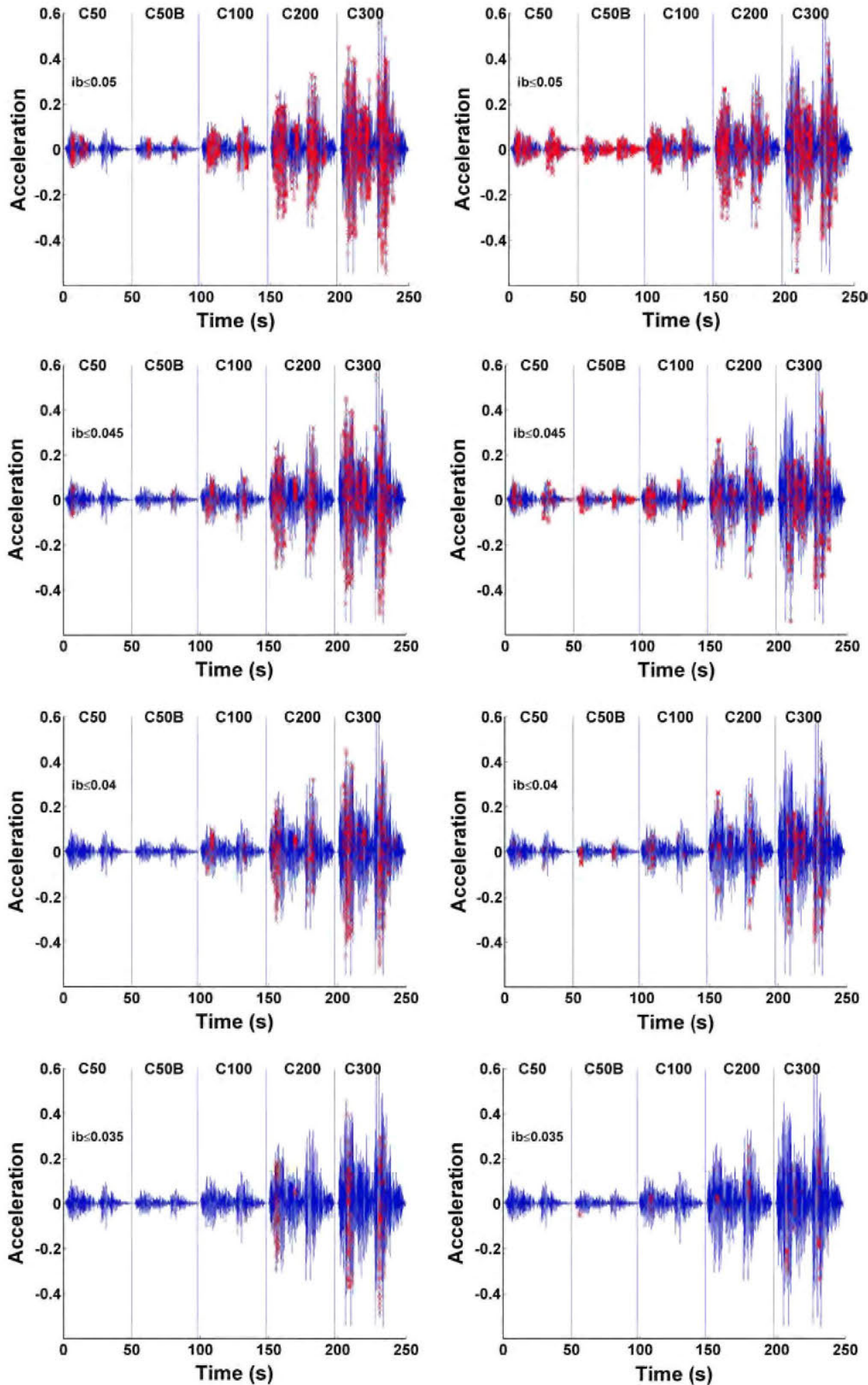

Figure 18 represents the Critical Events accumulated over the successive seismic simulations in both connections for different limits of the $b$-value and $i b$-value $(0.05,0.045,0.04$ and 0.035 ).
When the limit is set at 0.05 , a remarkable increase in the amount of Critical Events for the interior connection P4 is observed during the first three seismic simulations (C50, $\mathrm{C} 50 \mathrm{~B}, \mathrm{C} 100)$, suggesting the development of macro-cracks 
Fig. 18 History of critical events accumulated in both beam-column connections P3 and $\mathrm{P} 4$ along all seismic simulations. Left $b$-value. Right $i b$-value
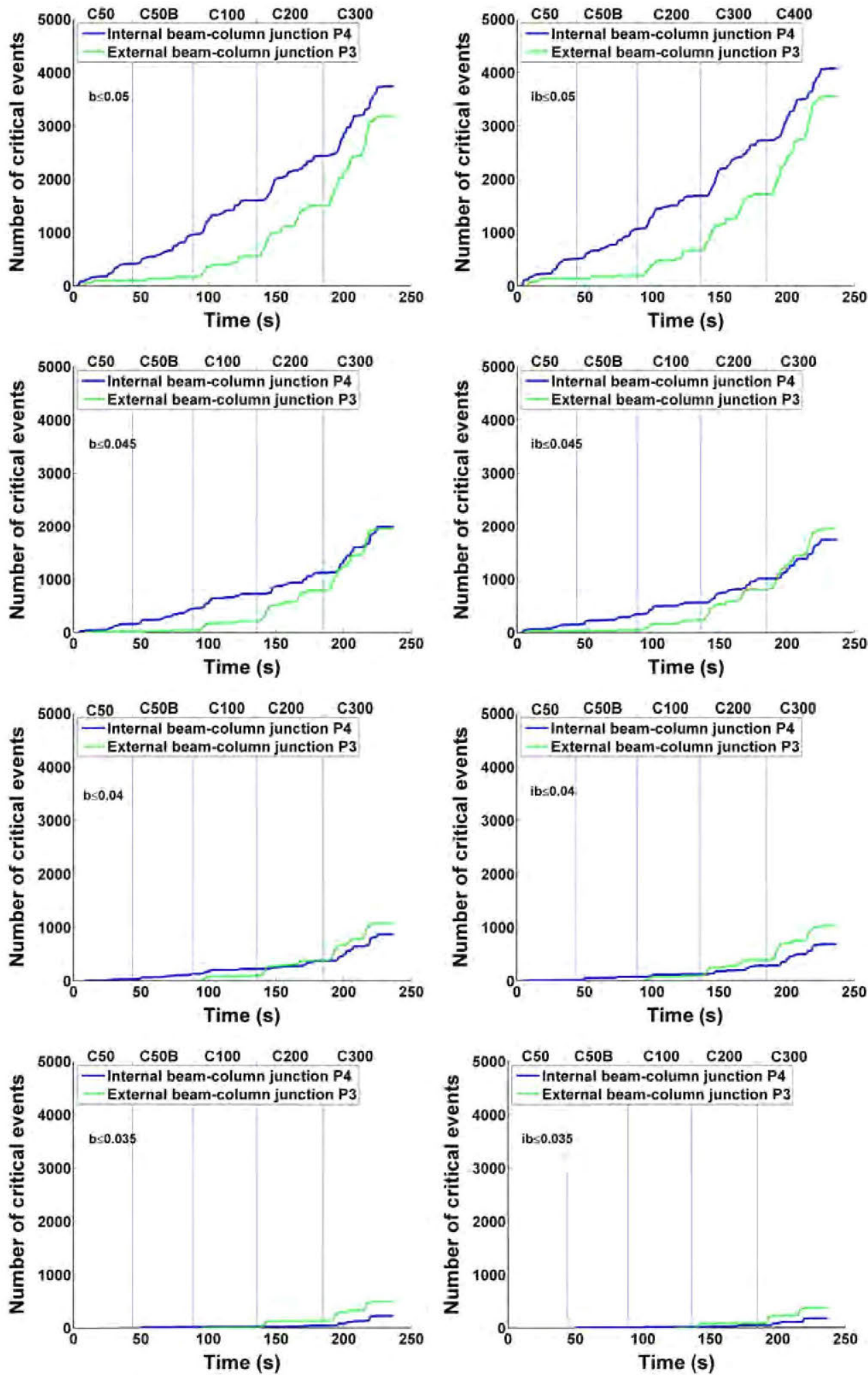

that were not observed during the tests. From then on, i.e. for seismic simulations $\mathrm{C} 200$ and $\mathrm{C} 300$, the rate of Critical Events significantly increases, suggesting an important increase of damage on the specimen. This result is especially noticeable for the exterior connection P3. When the limit is 0.045 , the number of Critical Events becomes lower than for 0.05 , but this number is still significant for seismic simulations $\mathrm{C} 50, \mathrm{C} 50 \mathrm{~B}$ and $\mathrm{C} 100$, thus contradicting the fact that no macro-cracks were observed during this seismic simulation. 
However, when the limit is set at 0.04 or 0.035 , two important changes can be observed: (i) few Critical Events are detected during the first seismic simulations ( $\mathrm{C} 50, \mathrm{C} 50 \mathrm{~B}$ and $\mathrm{C} 100$ ), which is in good agreement with the visual observation of no macro-cracks on the specimen; and (ii) more Critical Events are detected for exterior connection P3 than for the interior one, $\mathrm{P} 4$, during seismic simulations $\mathrm{C} 200$ and C300. This second observation is consistent with the fact that more macro-cracks were observed by the naked eye in the exterior connection than in the interior one.

These results suggest that the limit of 0.05 proposed in past research for evaluating macroscopic cracks in static or quasistatic tests is not suitable for dynamic tests, the value of 0.04 being more appropriate. This statement is supported below by comparing the $b$ and $i b$ values with: (i) another damage index, the IDI, commonly accepted as a good indicator of the level of damage endured by a reinforced concrete frame structure subjected to seismic actions; and (ii) with visual observation of the cracks.

\subsection{Comparison of $b$ and ib Values with IDI Index}

In a previous work [19], the so-called maximum Inter-story Drift Index (IDI) was calculated for this test specimen and for the seismic simulations described above. This index has traditionally been associated with the level of damage experienced by a structure subjected to lateral displacements due to a ground motion. However, a major limitation of this index is that it does not take into account the accumulated damage in the structure. The $I D I$ of the test specimen investigated here was calculated from the measurement provided by the displacement transducers (LVDTs) installed at each floor level.

The inter-story drift at a given instant $t$ is defined as the ratio of the relative displacement between the upper and lower floors of a given story $(\delta)$ to the height of the story $(h)$, and it is commonly expressed as a percentage. The $I D I$ is the maximum absolute value of this ratio during the seismic simulation, i.e.

$I D I=\max \left\{\frac{\delta}{h}\right\}$

The $I D I$ has been associated with different levels of damage for reinforced concrete frame structures, as shown in Table $1[19]$.

Table 2 shows the IDIs obtained in each of the seismic simulations during the shake-table tests. Based on these experimental values and the corresponding levels of damage shown in Table 1, the global damage to the structure after each seismic simulation can be summarized as follows. The structure remained basically elastic during seismic simulations C50 and $\mathrm{C} 50 \mathrm{~B}$, which is consistent with the fact that the reinforcing steel did not yield and only micro-cracks occurred
Table $1 I D I$ values and corresponding levels of damage

\begin{tabular}{ll}
\hline$I D I(\%)$ & Level of damage \\
\hline $0-0.5$ & No damage \\
$0.5-1.0$ & Moderate damage \\
$1.0-3.5$ & Severe damage \\
$>3.5$ & Very severe samage \\
\hline
\end{tabular}

Table $2 I D I$ obtained for each seismic simulation

\begin{tabular}{ll}
\hline Test & $I D I$ \\
\hline C50 & 0.22 \\
C50B & 0.24 \\
C100 & 0.5 \\
C200 & 1.2 \\
C300 & 7.9 \\
\hline
\end{tabular}

in the concrete. During seismic simulation C100 the structure remained basically undamaged; this is confirmed by the fact that minor yielding of the longitudinal reinforcement occurred (strains up to about 2 times the yield strain) and the beam end sections were on the brim of yielding. The test specimen suffered severe damage during seismic simulation C200, and very severe damage (near collapse) in seismic simulation C300.

\subsection{Crack identification with the Naked Eye}

A visual inspection of the test specimen at the end of each seismic simulation revealed the following pattern of macrocracks, drawn schematically in Fig. 19:

Seismic simulation C50 No visible cracks.

Seismic simulation C50B No visible cracks.

Seismic simulation C100 Some minor cracks.

Seismic simulation C200 Opening of new cracks and growth of previous cracks.

Seismic simulation C300 Large cracks were observed, coming from both the growth of previous ones and the formation of new cracks. The maximum width of these cracks was about $3 \mathrm{~mm}$; in some cases they were accompanied by a sudden vertical slide of approximately $10 \mathrm{~mm}$ between the two sides of the crack.

Figure 20 shows pictures of the specimen after the last test (C300). Large cracks in both beam-column connections can be observed.

Thus, the global level of damage assessed in terms of $I D I$ and the cracks identified after each seismic simulation support the limit value 0.04 for the $b$ - and $i b$-values proposed in this study for dynamic earthquake-type loadings, and confirm that for this type of loading the limit value 0.05 proposed in past studies on the basis of static or pseudo-static tests is not appropriate. 
Fig. 19 Pattern of macro-cracks observed after simulations: C100 (green), C200 (orange) and C300 (blue) (Color figure online)
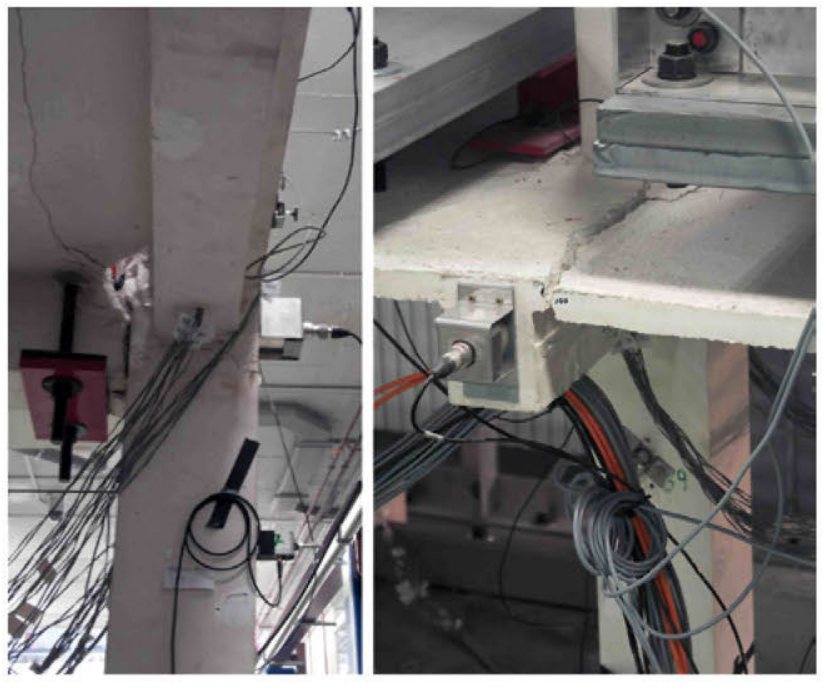

Fig. 20 Beam-column connections after seismic simulation C300. Left exterior beam-column connection P3. Right interior beam-column connection $\mathrm{P} 4$

\section{Conclusions}

The feasibility of using the $b$-value and $i b$-value calculated from $\mathrm{AE}$ measurements to assess the damage in $\mathrm{RC}$ structures subjected to earthquakes was investigated in this paper. By comparing the $b$-and $i b$-values with the actual damage observed in an RC frame tested on a shake-table, it was found that the 0.05 limit value that previous research associates with the onset of severe damage (i.e. the development and growth of macro-cracks in concrete) should be revised in the case of dynamic earthquake-type cyclic loadings. The global level of damage evaluated quantitatively in terms of the IDI, and qualitatively with the cracks identified after each seismic simulation, would support adopting a lower limit value, as proposed in this study for earthquake-type dynamic loadings.

Acknowledgments This research received financial support from the local government of Spain, Consejería de Innovación, Ciencia y Tecnología, Project P07-TEP-02610, from the FPU Program of the Education, Culture and Sports Ministry of Spain, and from the European Union (Fonds Européen de Dévelopment Régional). 